\title{
Homeopaths will be allowed to practise modern medicine in Maharashtra after one year pharmacology course
}

In this News story by Sanjeet Bagcchi (BMJ 2014;348:g422, doi:10.1136/bmj.g422) a comment in the last paragraph about the pharmacology of homeopathic and modern medicine was incorrectly attributed to Dev Raj Rai, senior vice president of the Indian Medical Association, when it should have been attributed to Swapan Jana, a health activist.
We apologise for this error, which occurred during the editing process and was not the fault of the article's author.

Cite this as: BMJ 2014;348:g1218

๑) BMJ Publishing Group Ltd 2014 